\title{
BMJ Open Use and impact of point-of-care ultrasonography in general practice: a prospective observational study
}

\author{
Camilla Aakjær Andersen (10 , ${ }^{1}$ John Brodersen, ${ }^{2,3}$ Annette Sofie Davidsen, ${ }^{2}$ \\ Ole Graumann, ${ }^{4}$ Martin Bach B Jensen (10 ${ }^{1}$
}

To cite: Aakjær Andersen C, Brodersen J, Davidsen AS, et al. Use and impact of pointof-care ultrasonography in general practice: a prospective observational study. BMJ Open 2020;10:e037664. doi:10.1136/ bmjopen-2020-037664

- Prepublication history for this paper is available online. To view these files, please visit the journal online (http://dx.doi. org/10.1136/bmjopen-2020037664).

Received 11 February 2020 Revised 12 June 2020 Accepted 30 July 2020

Check for updates

(c) Author(s) (or their employer(s)) 2020. Re-use permitted under CC BY-NC. No commercial re-use. See rights and permissions. Published by BMJ.

${ }^{1}$ Center for General Practice, Aalborg University, Aalborg, Denmark

${ }^{2}$ Research Unit for General Practice and Section of General Practice, Department of Public Health, Faculty of Health Sciences, University of Copenhagen, Copenhagen, Denmark

${ }^{3}$ Primary Health Care Research Unit, Zealand Region, Copenhagen, Denmark ${ }^{4}$ Department of Radiology, Radiological Research and Innovation Unit, Odense University Hospital, Odense, Denmark

Correspondence to Dr Camilla Aakjær Andersen; caakjaer@dcm.aau.dk

\section{ABSTRACT}

Objectives To describe how general practitioners (GPS)

use point-of-care ultrasonography (POCUS) and how it influences the diagnostic process and treatment of patients.

Design Prospective observational study using an online questionnaire before and after POCUS.

Setting Office-based general practice.

Participants Twenty GPs consecutively recruited all patients examined with POCUS in 1 month.

Primary and secondary outcome measures We estimated the use of POCUS through the indication for use, the frequency of use, the time consumption, the extent of modification of the examination and the findings.

The influence on the diagnostic process was estimated through change in the tentative diagnoses, change in confidence, the ability to produce ultrasound images and the relationship between confidence and organs scanned or tentative diagnoses.

The influence of POCUS on patient treatment was estimated through change in plan for the patient, change in patient's treatment and the relationship between such changes and certain findings.

Results The GPs included 574 patients in the study. POCUS was used in patient consultations with a median frequency of 8.6\% (IQR: 4.9-12.6). Many different organs were scanned covering more than 100 different tentative diagnoses. The median time taken to perform POCUS was 5 min (IQR: 3-8). Across applications and GPs, POCUS entailed a change in diagnoses in $49.4 \%$ of patients; increased confidence in a diagnosis in $89.2 \%$ of patients; a change in the management plan for $50.9 \%$ of patients including an absolute reduction in intended referrals to secondary care from $49.2 \%$ to $25.6 \%$; and a change in treatment for $26.5 \%$ of patients.

Conclusions The clinical utilisation of POCUS was highly variable among the GPs included in this study in terms of the indication for performing POCUS, examined scanning modalities and frequency of use. Overall, using POCUS altered the GPs' diagnostic process and clinical decisionmaking in nearly three out of four consultations.

Trial registration number NCT03375333.

\section{INTRODUCTION}

Point-of-care ultrasound (POCUS) is used in general practice in several countries. ${ }^{1-4}$ A recent systematic review found that few

\section{Strengths and limitations of this study}

This study explores the use of point-of-care ultrasound in a broader sample of general practitioners (GPs).

- The study was developed through a comprehensive qualitative work and designed to mimic daily practice.

- This study may be subject to selection bias since the participating GPs likely constitute a subset of physicians with a special interest in ultrasonography.

- The study registrations were time consuming and fewer patients than expected were included.

- Point-of-care ultrasound changed diagnosis, plan and/or treatment for most patients, but we did not evaluate whether these changes improved or worsened patient care.

studies described the use of POCUS in the hands of the general practitioner (GP) and that obstetric, abdominal and heart examinations were the most commonly described. ${ }^{5}$ The included studies, however, focused on selected scanning modalities and largely aimed to explore a possible transition of ultrasound examinations from secondary to primary care. The recent introduction of POCUS, as something disparate from ultrasound examinations performed by radiologists or other highly specialised physicians, ${ }^{67}$ prepares the ground for more widespread use, as it encourages clinicians to apply POCUS as part of the physical examination of patients. ${ }^{89}$ Hence, the current use of POCUS in general practice may differ from use previously reported. No previous studies have quantified the use of POCUS in a larger group of GPs with different types of ultrasound training, who have adopted the technology without either constraining or supporting guidelines and without financial incentives.

Evidence from the secondary healthcare sector has shown that certain POCUS applications affect the diagnostic process leading to earlier and more correct diagnosis, ${ }^{10}{ }^{11}$ 
a subsequent change in patient treatment and a more rational use of healthcare resources. ${ }^{12}{ }^{13} \mathrm{~A}$ few recent studies from general practice suggest the same. ${ }^{14-16}$ However, little attention has been given to the specific impact of POCUS on the diagnostic process in general practice and GPs' clinical decision-making.

The aim of this study was to describe how GPs use POCUS in their daily practice and how it influences the diagnostic process and the treatment of patients.

\section{METHOD}

\section{Study design}

We report the study findings according to Strengthening the Reporting of Observational Studies in Epidemiology guidelines.

\section{Study setting}

The study was conducted in office-based general practices in Denmark, where GPs were already using POCUS. Denmark has universal, publicly funded healthcare system, where almost all patients are registered with a GP. The GPs act as gatekeepers for secondary care services including ultrasonography. GPs receive no fee for performing POCUS in primary care.

\section{Participating GPs}

Twenty GPs were recruited through POCUS networks, conferences and teaching sessions (online supplementary appendix 1).

To be included in the study, GP had to:

- Have used POCUS for a more than 6 months.

- Use POCUS for a minimum of two anatomical areas.

- Use POCUS on a daily basis.

- Have participated in formalised POCUS training, for example, an ultrasound course.

- Work in a practice with a patient population over 1400.

- Work in the practice minimum 4 days a week.

GPs with an ultrasound system more than 10 years old or with any possible financial conflict of interest were excluded. The GPs were enrolled in the study stepwise from January 2018 to August 2018 to account for any seasonal variation in POCUS examinations. Prior to the study, participating GPs' POCUS competences were assessed using a modified version of the Objective Structured Assessment of Ultrasound Skills ${ }^{17}$ and an objective structured clinical examination evaluation sheet (online supplementary appendix 2). The GPs were blinded to the results of this assessment.

\section{Participating patients}

All patients who sought care for conditions that the participating GP found relevant for POCUS examination were invited to participate in the study.

\section{Data collection and study procedure}

The GPs consecutively registered information on all POCUS examinations during 1 month (20-25 working days), while performing POCUS according to their usual indications and standards and using their own ultrasound systems. When a GP planned to use POCUS, the patient received study information and a written informed consent was obtained. Thereafter, the GPs accessed an online SurveyXact questionnaire (Rambøll, Aarhus, Denmark) and completed items before and after conducting POCUS. A time log measured the time between the before and the after POCUS registrations (see online supplementary appendix 3 for questionnaire details).

We also registered the total number of face-to-face patient consultations during the study period and the number of eligible patients who were not included due to, for example, time constraints. The primary investigator (CAA) visited the GPs' clinics on the first day of inclusion to help with the registrations and to perform a validity test of the GPs' registration.

\section{Outcome measures}

The use of POCUS in general practice was estimated through: (1) the indication for using POCUS; (2) the frequency of POCUS; (3) the time consumption for POCUS; (4) the extent of modification of the POCUS; and (5) the POCUS findings.

The influence of POCUS on the diagnostic process in general practice was estimated through: (1) change in the tentative diagnoses according to the international classification of primary care second edition (ICPC2) $^{18}$ before and after POCUS; (2) the GP's declared change in confidence in the main tentative diagnosis after the use of POCUS; (3) the GP's ability to technically produce ultrasound images; (4) the relationship between confidence in the main tentative diagnosis and the examined scanning modalities, reduction in the number of tentative diagnoses and change from symptom to disease-specific diagnoses.

The influence of POCUS on patient treatment in general practice was estimated through: (1) change in plan for the patient; (2) change in patient's treatment; (3) the relationship between certain findings and changes in the management plan or treatment of the patient.

\section{Sample size and statistical analysis}

Based on a questionnaire study, ${ }^{3}$ we estimate that there were around 75 GPs in Denmark, who would meet our inclusion criteria. We found it realistic to include 20 of the GPs in the study. Based on an interview study with Danish GPs, ${ }^{16}$ we estimated that the GPs would use POCUS 2-3 times a day. Assuming a participation rate of $80 \%$, we expected to include 640-960 patients during the study period of 1 month.

Data were analysed using STATA V.15.0 (StataCorp) according to a predefined analysis plan. Categorical variables were summarised using absolute frequencies and continuous variables using mean and standard deviation (median and IQR if not normally distributed). Relative-risk reduction in referrals for secondary care was 
calculated by considering referrals as events, the beforePOCUS registrations as controls and the after-POCUS registrations as interventions. Our predefined hypotheses, all published in clinical trials, about the relationship between variables were analysed using Fisher's exact test and a significance level of 0.05 .

\section{Patient and public involvement statement}

Written informed consent was obtained from all participating GPs and patients and all data were pseudoanonymised using deidentification numbers. Only the principal investigator (CAA) knew the identity of the GPs and only the GPs knew the identity of the participating patients. Patients were involved and invited to provide feedback during the design and pilot testing of the registration tools used in this study.

\section{RESULTS}

Twenty GPs from 18 clinics each enrolled a median of 26 (IQR 17-40) patients. Data from 574 patients were available for analysis, and in 528 patients data were available for before-after comparison (figure 1). Background characteristics are given in table 1.

POCUS competences were assessed in 19 GPs covering between two and six applications, depending on their normal use of POCUS (figure 2 and online supplementary appendix 2).

\section{The use of POCUS}

Each GPs performed between 12 and 84 POCUS examinations (median: 32.0 (IQR: 17.8-42.8)) corresponding to an individual average between 0.6 and 3.9 ultrasound examinations per day. The GPs had between 13.0 and 24.4 face-to-face patient consultations per day (median: 15.9 (IQR: 14.2-17.8)). Hence, during the study period, each GPs performed POCUS in between $3.7 \%$ and $20.8 \%$ of all face-to-face consultations (median: 8.6 (IQR: 4.9-12.6)).

When GPs were using POCUS, they aimed primarily to confirm or disconfirm a specific clinical condition (73.1\%), or to explore the reason for the patient's symptoms without having a specific clinical condition in mind $(20.2 \%)$, but they rarely planned to do both $(1.6 \%)$. A total of 126 different ICPC2 codes were registered as the primary tentative diagnosis before the use of POCUS (online supplementary appendix 4).

POCUS was used to examine many different organs and structures (figure 3). The GPs registered examining a total of 834 scanning modalities in 570 POCUS examinations (data missing in four patients); most commonly heart and lung in combination and different combinations of abdominal organs. In addition, we found that GPs modified their POCUS examination to include more scanning modalities than intended in $15.5 \%$, and fewer scanning modalities than intended in $8.0 \%$ of all ultrasound examinations.

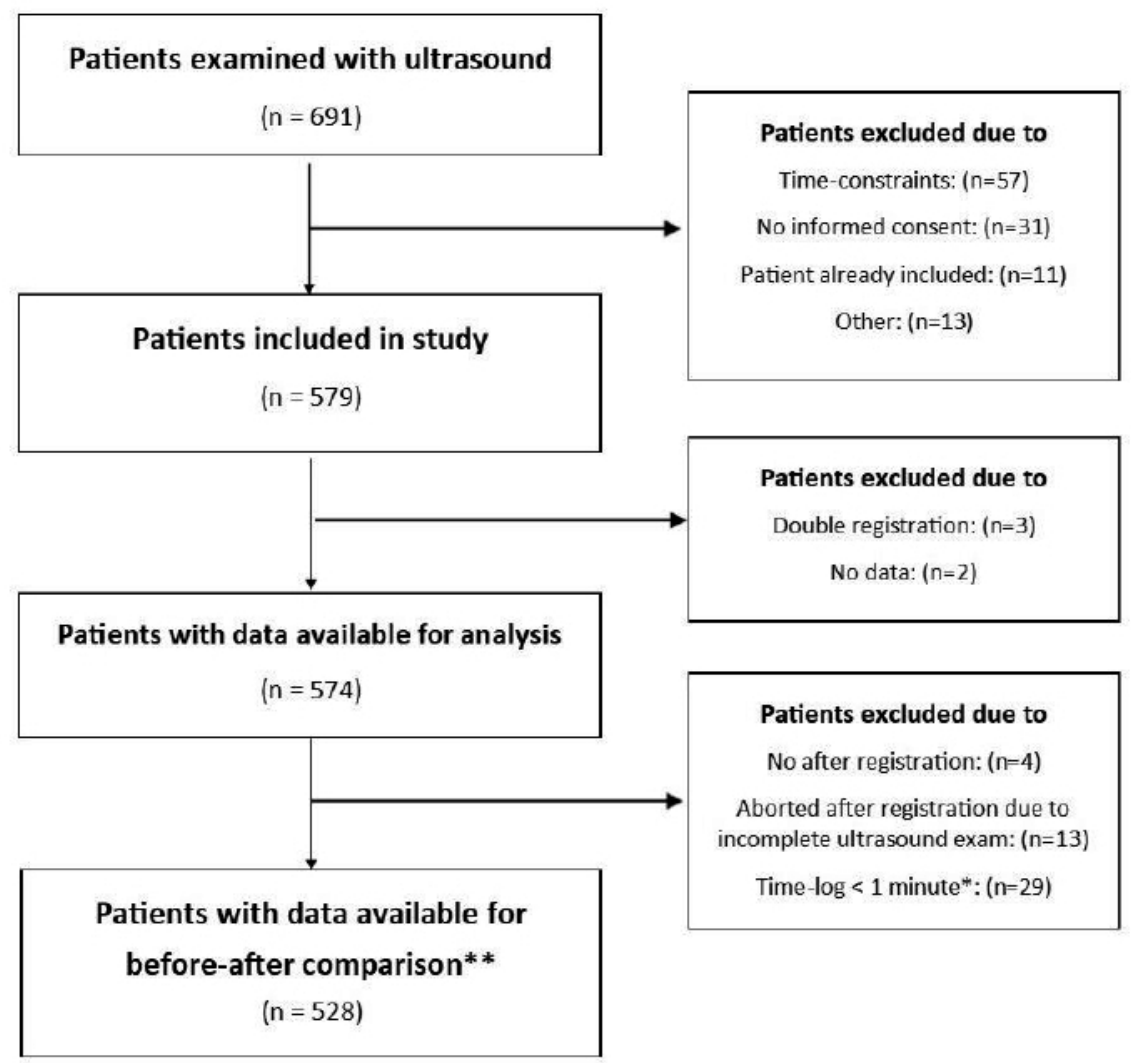

Figure 1 Patient flow diagram. ${ }^{*}$ Time-log $<1 \mathrm{~min}=$ no separation between before and after registration in the questionnaire. Before registration was deleted. ${ }^{* \star}$ We had 545 before registrations, 557 after registrations and 528 complete before and after registrations. 
Table 1 Background characteristics

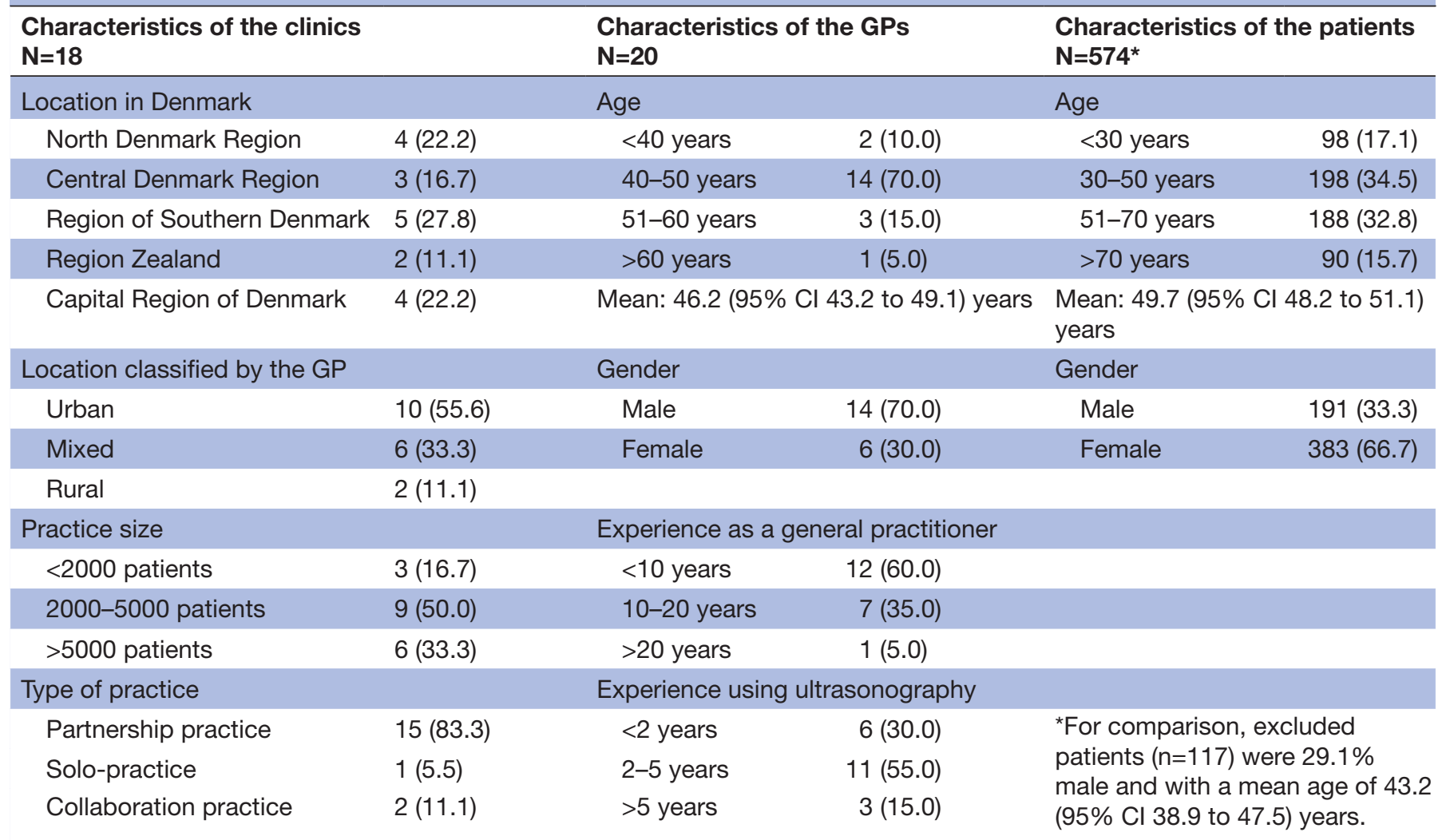

Number (percentage) of the total number of participants in each group (N).

The median time consumption for the POCUS examination was $5 \mathrm{~min}$ (IQR: 3-8) but varied from 1 to $30 \mathrm{~min}$ (figure 3).

Images of the relevant structures could be produced in between $95 \%$ and $100 \%$ of the applications, though some seemed to cause more difficulty: lymph nodes $(75 \%)$; pancreas $(75 \%)$; ovaries $(78 \%)$; heart $(89 \%)$; kidney $(93 \%)$; and others $(86 \%)$.

The GPs classified their POCUS examinations to include: certain positive findings $(45.7 \%)$; uncertain positive findings $(9.3 \%)$; certain negative findings $(32.3 \%)$; uncertain negative findings $(10.2 \%)$; and different combinations of these findings $(1.7 \%)$. In addition, the GPs registered incidental findings in six patients.

\section{POCUS influence on the diagnostic process}

POCUS changed the main tentative diagnosis in $49.4 \%$ of consultations (table 2 and online supplementary appendix 4). This encompassed a reduction in the number of patients where the GP had more than one tentative diagnosis from $29.6 \%$ before to $17.5 \%$ after the POCUS examination. There was also a reduction in the number of symptom diagnoses and a corresponding increase in the number of disease-specific, infectionrelated, cancer-related and emergency-related diagnoses after as compared with before the POCUS examination (online supplementary appendix 5).
After POCUS, the GPs declared the following change in their confidence in the primary tentative diagnosis: highly increased confidence $(60.5 \%)$; increased confidence $(28.5 \%)$; unchanged confidence $(6.6 \%)$; reduced confidence $(1.0 \%)$; and highly reduced confidence $(0.1 \%)$. Seven consultations entailed reduced confidence, the applications in these examinations were: heart (1), lung (1), thyroid and lymph nodes (1), subcutaneous process (1), gallbladder, liver and pancreas (1), tendon (1) and uterus (1). Increased confidence did not seem to depend on area of POCUS application as we found no variation beyond what could be expected by chance (0.082). Likewise, no relationship was found between a reduction in the number of tentative diagnosis and increased confidence $(\mathrm{p}=0.127)$. We did, however, find a relationship between increased confidence and a change from symptom diagnosis to disease-specific diagnosis $(\mathrm{p}=0.037)$.

\section{POCUS influence on patient treatment}

POCUS changed the intended management plan for patients in $50.9 \%$ of consultations (table 2), including a reduction in the absolute number of patients referred to hospital or secondary care clinics from $174(33.0 \%)$ to $105(19.9 \%)$ patients, and a reduction in the number of patients referred for imaging in the secondary sector from $86(16.3 \%)$ to $30(5.7 \%)$. Overall, there was an absolute reduction in intended referrals for secondary care 


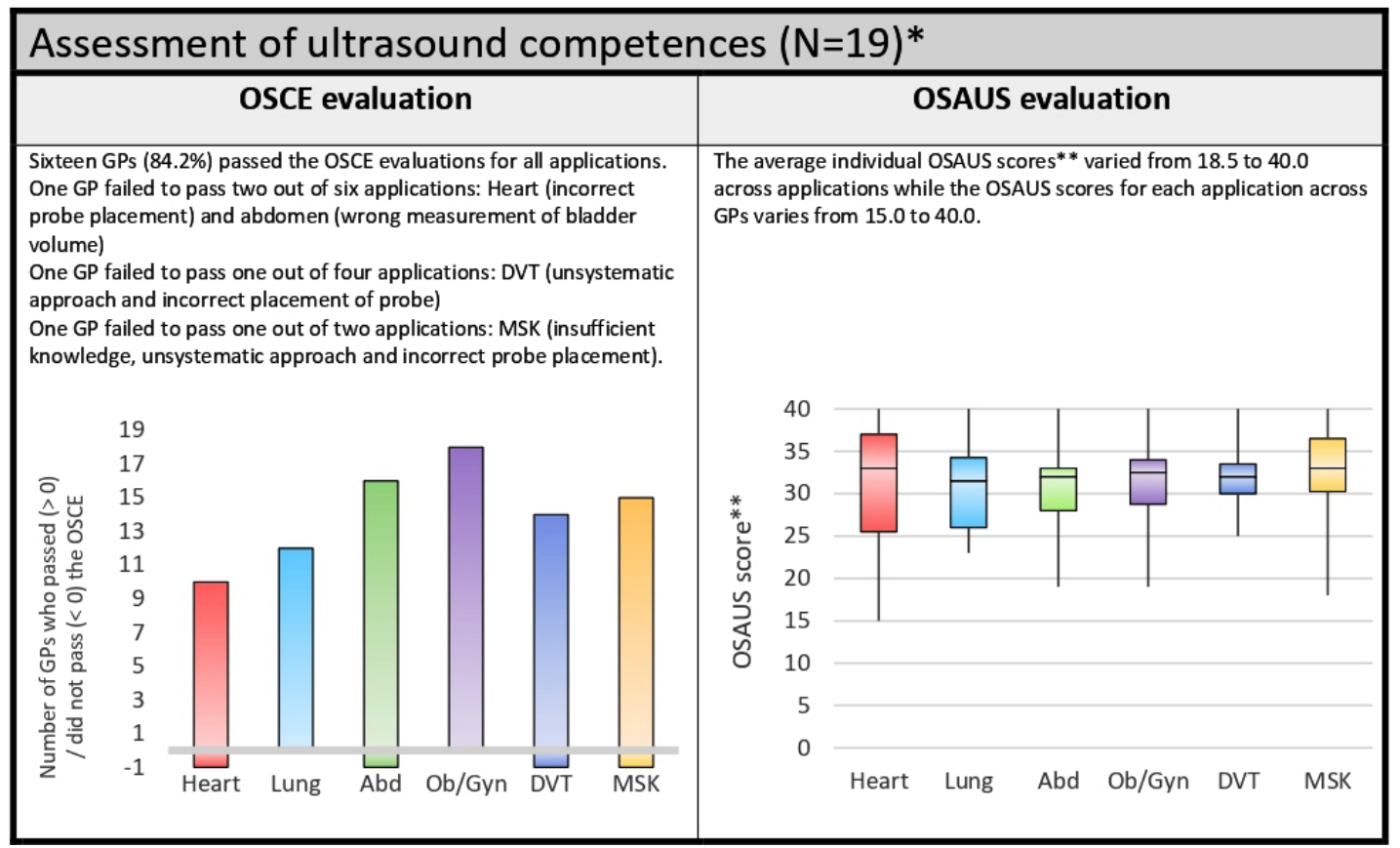

Figure 2 Ultrasound competences of the participating general practitioners (GPs). *A teacher in point-of-care ultrasonography (PoC-US) and radiology specialist (OG) assessed 19 of the GPs' performances in a standardised setting using an adapted version of ageneric ultrasound rating scale (The Objective Structured Assessment of Ultrasound Skills (OSAUS) ${ }^{17}$ ) and asked questions about the examination according to an objective structured clinical examination (OSCE) evaluation sheet. The GPs were asked to demonstrate PoC-US according to their usual routine and they were only assessed in the applications that they normally used. One GPs declined to participate in this evaluation. ${ }^{* *}$ OSAUS assessed on a scale from 0 to 40 . Abd, abdomen; DVT, deep venous thrombosis; MSK, musculoskeletal; Ob/Gyn, obstetric and gynaecological.

from $49.2 \%$ to $25.6 \%$ corresponding to an absolute risk reduction of $23.6 \%$ and a relative-risk reduction of $48.0 \%$. Correspondingly, the number of patients with planned follow-up in primary care increased from 185 (35.0\%) to $215(40.7 \%)$, and patients with no planned follow-up increased from $106(20.1 \%)$ to $198(37.5 \%)$ following POCUS (table 3).

After POCUS, the intended treatment was changed in $26.5 \%$ of consultations (table 2 ). The number of patients planned for referral to treatment in the secondary sector fell from $87(16.4 \%)$ to $63(11.9 \%)$. The number of patients where the GP would not initiate treatment fell from $283(53.6 \%)$ to $269(50.9 \%)$, whereas the number of patients where the GP initiated treatment increased from $168(31.8 \%)$ to 208 (39.4\%; table 3). We found no relationship between the GPs' classification of certain findings and a change in the patient's plan $(\mathrm{p}=0.913)$, or change in the patient's treatment $(p=0.214)$.

Overall change as a result of POCUS (change in diagnosis and/or change in the patient's plan and/or change in the patient's treatment) was found in $71.8 \%$ of consultations (table 2).

\section{DISCUSSION}

\section{Summary of main findings}

This study showed that across applications POCUS had a large impact on the diagnostic process in general practice. POCUS changed the tentative diagnoses in $49.4 \%$ of patients and increased confidence in the main tentative diagnosis in $89.2 \%$ of patients. POCUS changed the intended management plan in $50.9 \%$ of patients, including a relative-risk reduction in planned referrals of $48 \%$, and a change in the intended treatment of $26.5 \%$ of patients.

\section{Strengths and limitations}

This study had a broader sample of scanning GPs than most reported studies. Furthermore, the registration tool was developed through comprehensive qualitative work ${ }^{16}$ and pilot testing. We designed the study to mimic daily work and to avoid recall bias in the registrations. Furthermore, the GPs were blinded to the results of their competence evaluations.

We included fewer patients than expected. The patient information and study registrations added $10 \mathrm{~min}$ to the consultation and, due to time constraints, GPs may have chosen not to perform some POCUS examinations. Hence, the frequency of POCUS reported in this study may be underestimated.

The participating GPs varied considerably in their background characteristics, but given their interest in POCUS, they most likely constitute a select group compared with a broader population of GPs. The participating GPs resembled the general GP population in Denmark in terms of the location and size of the clinic, but not in terms of age, gender or organisation of the clinic. Specifically, the participants were younger, more often men and more 


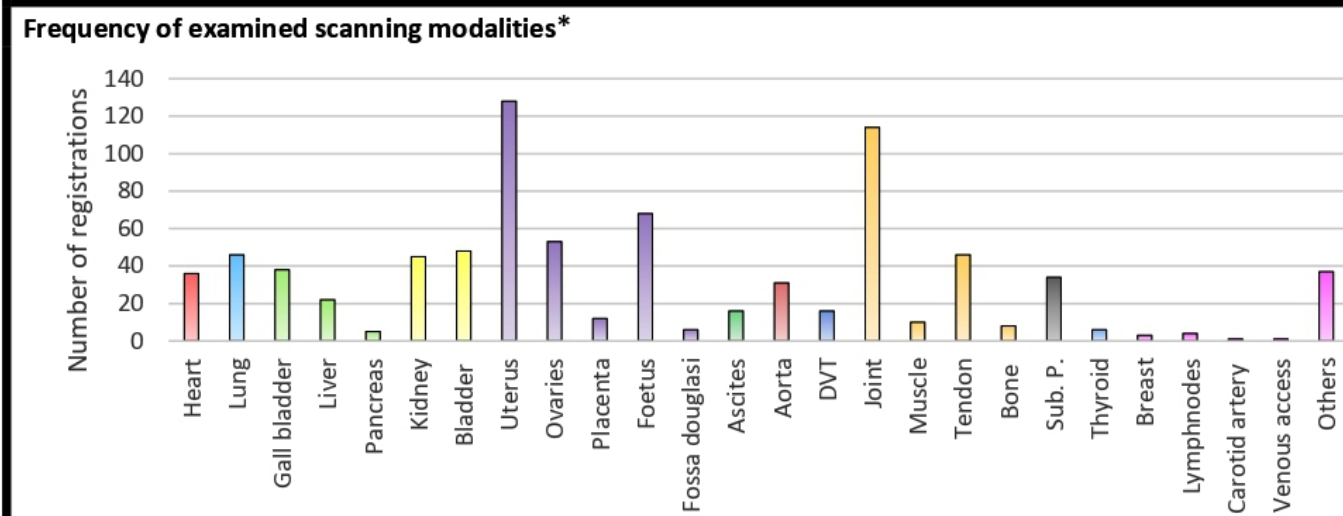

Frequency of exams including different applications**

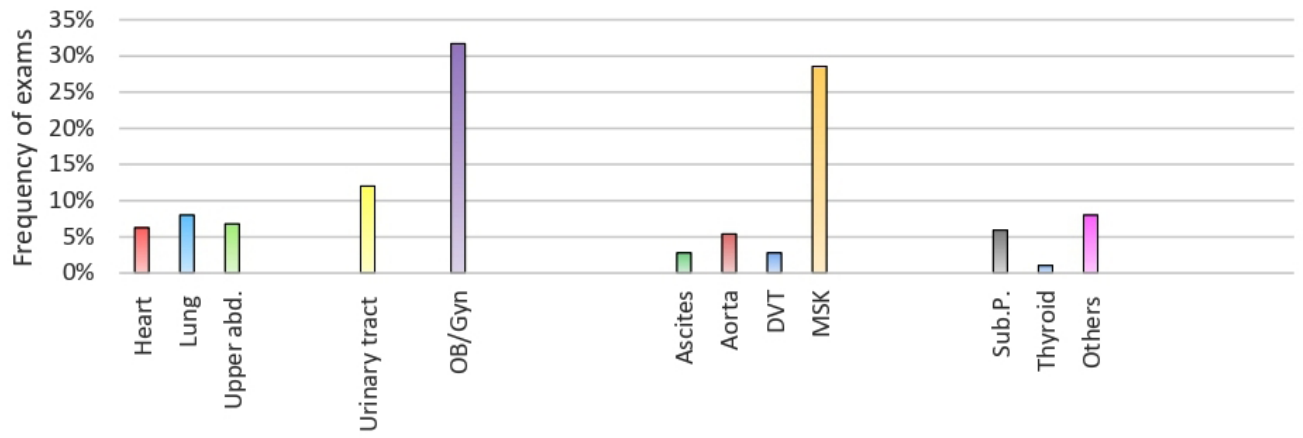

Time consumption per application***

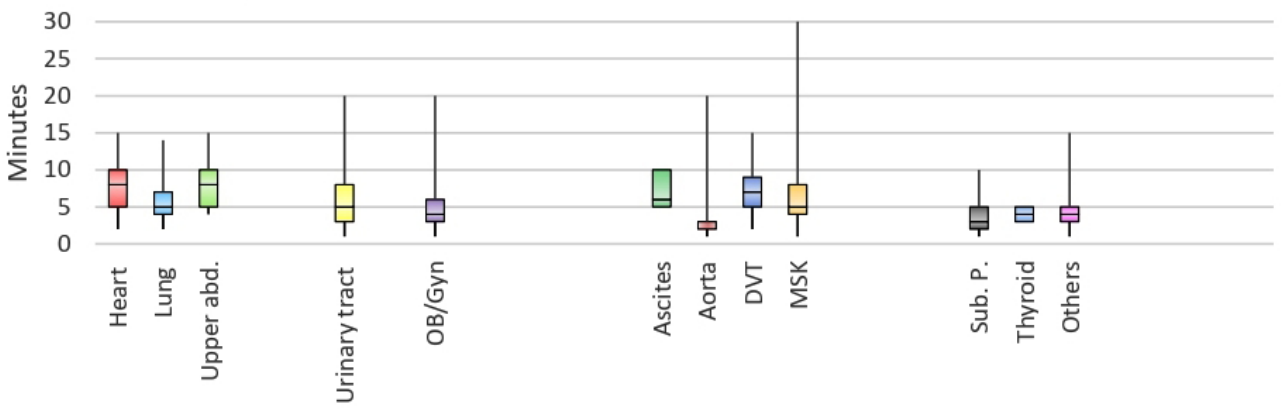

Figure 3 Use of ultrasonography in general practice. *After registrations of scanning modalities $(\mathrm{N}=834)$. ${ }^{* \star} \mathrm{Number}$ of exams with an after registration of scanning modalities $(\mathrm{N}=574)$. The registered scanning modalities are categorised according to application: Upper abd.= upper abdominal organs (including liver, gall bladder, pancreas), urinary tract (including kidney, and bladder); OB/Gyn=obstetric and gynaecological (including uterus, ovaries, placenta, fetus and fossa douglasi); Ascites= scans for abdominal flee fluid; DVT= scans fordeep venous thrombosis; MSK= musculoskeletal (including joints, muscle, tendon, bone and joint puncture); Sub.P.= Subcutaneous process. The others category includes free text answers and registered applications with a frequency below five examinations: intestines incl. appendix andrectum $(\mathrm{N}=7)$, bursa $(\mathrm{N}=6)$, unclassified abdominal structures $(\mathrm{N}=6)$, testis $(\mathrm{N}=5)$, amnion fluid $(\mathrm{N}=4)$, lymph nodes $(\mathrm{N}=4)$, breast $(\mathrm{N}=3)$, soft tissue $(\mathrm{N}=2)$, hernia $(\mathrm{N}=2)$, ureter $(\mathrm{N}=1)$, Larynx $(N=1)$, varicose vein $(N=1)$, unclassified abscess $(N=1)$, carotid artery $(N=1)$, blood vein for venous access $(N=1)$ and unclassified structure on finger $(\mathrm{N}=1)$. ${ }^{* \star *}$ Time registration if examination only included one application $(\mathrm{N}=486)$. Described as median time consumption, IQR and range.

often working in a partnership practice. ${ }^{19}$ Being a selected group of early adapters of the technology, it is plausible that the participating GPs rely heavily on POCUS in their daily work and subsequently that the frequency of increased confidence and change in diagnosis, plan or treatment is higher in this particular group of GPs.

We used Fisher's exact test to explore overall associations. Due to the lack of statistical power, however, no firm conclusions can be made regarding the relationship between the GP's confidence in the main tentative diagnosis and the organs scanned.

\section{Findings in context}

In line with previous research, ${ }^{25}{ }^{20}$ we found large variation in the application of POCUS in general practice. The most common applications in this study were pelvic and musculoskeletal POCUS. This may be explained by the fact that all participating GPs had received training 
Table 2 Change in diagnosis, management plan or treatment following the use of point-of-care ultrasonography (POCUS)

\begin{tabular}{|c|c|c|c|c|c|}
\hline POCUS application* & $\mathbf{N}$ & $\begin{array}{l}\text { Change in the } \\
\text { tentative diagnoses, } \\
\mathrm{n}(\%)\end{array}$ & $\begin{array}{l}\text { Change in the intended } \\
\text { management plan } \\
\mathrm{n}(\%)\end{array}$ & $\begin{array}{l}\text { Change in the } \\
\text { intended treatment } \\
\mathrm{n}(\%)\end{array}$ & $\begin{array}{l}\text { Overall change†, } \\
\text { n (\%) }\end{array}$ \\
\hline Heart & 34 & $23(68)$ & 20 (59) & $10(29)$ & $29(85)$ \\
\hline Lung & 44 & $26(59)$ & $23(52)$ & $15(34)$ & $37(84)$ \\
\hline Upper abdomen & 36 & $22(61)$ & $17(47)$ & $11(31)$ & $25(69)$ \\
\hline Urinary tract & 67 & $41(61)$ & $35(52)$ & $20(30)$ & $50(75)$ \\
\hline $\begin{array}{l}\text { Obstetric and } \\
\text { gynaecological }\end{array}$ & 165 & $61(37)$ & $83(50)$ & $35(21)$ & 97 (59) \\
\hline Ascites & 15 & $10(67)$ & $9(60)$ & $8(53)$ & $10(67)$ \\
\hline Aorta & 29 & $25(86)$ & $11(38)$ & $5(17)$ & $26(90)$ \\
\hline Deep vein thrombosis & 13 & $10(77)$ & $10(77)$ & 4 (31) & 12 (92) \\
\hline Musculoskeletal & 157 & $76(48)$ & $90(57)$ & $55(35)$ & $124(79)$ \\
\hline Subcutaneous process & 31 & $16(52)$ & $18(58)$ & $10(32)$ & $22(71)$ \\
\hline Thyroid & 6 & $4(67)$ & $1(17)$ & $1(17)$ & $5(83)$ \\
\hline Other & 40 & $21(53)$ & $18(45)$ & $7(18)$ & $26(65)$ \\
\hline Total & 528 & 261 (49) & 269 (51) & 140 (27) & 379 (72) \\
\hline
\end{tabular}

*The following registered scanning modalities are categorised according to POCUS application: upper abdominal organs (including liver, gall bladder, pancreas), urinary tract (including kidney, and bladder), obstetric and gynaecological (including uterus, ovaries, placenta, fetus and fossa douglasi), musculoskeletal (including joints, muscle, tendon, bone, and joint puncture). The others category includes free text answers and registered applications with a frequency below five examinations.

†Overall change includes change in either diagnoses, management plan and/or treatment.

in pelvic POCUS and all but two had participated in a musculoskeletal POCUS course. Another explanation may relate to patient encounters in Danish general practice. A previous qualitative study has described how Danish GPs perform POCUS examinations that they consider relevant in their patient population ${ }^{16}$ and musculoskeletal conditions are the most common organspecific complaints raised by patients in Danish general practice. ${ }^{21}$ Moreover, in a recent needs assessment, pelvic ultrasound was found to be the POCUS application GPs had most interest in learning. ${ }^{22}$

This study illustrates that POCUS is used to support the physical examinations of patients presenting with a very broad range of clinical conditions. There have been attempts to outline which POCUS examinations are best suited to general practice. ${ }^{4022-25}$ The evidence base for these attempts is sparse, however, and there may be significant differences between countries regarding which examinations are most relevant. ${ }^{3}$ In addition, some examinations may be easier to master than others, ${ }^{5}$ both in terms of achieving competence and in terms of maintaining competence over time. The latter may be particularly important in general practice where the frequency of some POCUS examinations is as low as shown in this study (figure 3). Some studies have reported high diagnostic accuracies of GPs' POCUS examinations, when these were compared with repeated scans by imaging specialists. ${ }^{15} 26$ However, these studies only included few scanning modalities, a rather small number of GPs, and the evaluation of accuracy was made shortly after participation in a training programme. Hence, we do not know if the results would be equally good if POCUS was applied for more applications, in a wider selection of GPs, or if long-term proficiency is achievable. Determining whether POCUS use in general practice results in better patient outcomes should include an evaluation of both the diagnostic accuracy (including potential overdiagnosis) of the performed examinations as well as the medical decision-making following the scan. In our baseline evaluation of the GPs scanning competences, we found that a few of the GPs lacked the practical skills for performing the scans, despite using POCUS regularly and having participated in training (figure 2). Likewise, we found that the GPs described their POCUS findings as uncertain in $19.7 \%$ of examinations. Officebased GPs may be used to navigating in uncertainty and performing up to a certain level before referring patients on to more advanced care. Still, POCUS is a particularly user-dependent technology ${ }^{23} 27$ and the ability to rule in or rule out, as well as the prevalence and interpretation of incidental findings, may differ between applications. ${ }^{528}$ Thus, there is a need for more research and evidencebased guidelines to support GPs in choosing what to scan and how to integrate findings into clinical care.

Previous studies from hospitals have shown that some POCUS examinations entail a change in patient care $^{10} 1129-31$ and our study suggests that this finding also applies in primary care. The GPs' registration data showed that $49.2 \%$ of patients would have had onward referral if POCUS had not been available. This referral 
Table 3 Registered change in patient care following the use of point-of-care ultrasonography

\section{Before After registrations registrations \\ n n}

\begin{tabular}{|c|c|c|}
\hline \multicolumn{3}{|c|}{ Change in the intended plan for the patient } \\
\hline $\begin{array}{l}\text { Acute admission to } \\
\text { hospital }\end{array}$ & 10 & 12 \\
\hline $\begin{array}{l}\text { Subacute referral to } \\
\text { hospital }\end{array}$ & 32 & 16 \\
\hline $\begin{array}{l}\text { Elective referral } \\
\text { hospital }\end{array}$ & 50 & 32 \\
\hline $\begin{array}{l}\text { Subacute referral to } \\
\text { specialist }\end{array}$ & 18 & 7 \\
\hline $\begin{array}{l}\text { Elective referral to } \\
\text { specialist }\end{array}$ & 64 & 38 \\
\hline Referral to radiology & 86 & 30 \\
\hline $\begin{array}{l}\text { Other referral } \\
\text { for example, } \\
\text { physiotherapist } \\
\text { (primary care services) }\end{array}$ & 20 & 32 \\
\hline Follow-up in the clinic & 165 & 183 \\
\hline No plan for follow-up & 106 & 198 \\
\hline \multicolumn{3}{|c|}{ Change in the intended treatment of the patient } \\
\hline I will initiate medication & 104 & 107 \\
\hline I will refer for treatment & 87 & 68 \\
\hline $\begin{array}{l}\text { I will initiate other } \\
\text { treatment }\end{array}$ & 64 & 115 \\
\hline $\begin{array}{l}\text { I will not initiate } \\
\text { treatment }\end{array}$ & 283 & 277 \\
\hline Other & $4^{*}$ & $11^{*}$ \\
\hline
\end{tabular}

Comparison between complete before and after registrations $(\mathrm{N}=528)$

The questions was designed as multiple choice. Results are provided as the number of registrations.

${ }^{*}$ Referral for treatment in physiotherapy.

frequency was reduced from $49.2 \%$ to $25.6 \%$ by using POCUS, whereas the number of patients with planned follow-up in general practice, or no follow-up, increased. Previous studies from general practice have suggested a reduction in referrals, ${ }^{14} 32$ but how POCUS in general practice affects overall healthcare costs is unknown.

\section{Implications for practice}

POCUS was used in in the patient consultation with a median of $8.6 \%$ and with a median time consumption of $5 \mathrm{~min}$. Hence, the use of POCUS is feasible in general practice despite differences in ultrasound equipment, experience, educational background and choice of examinations. POCUS largely impacted diagnostic certainty and patient management. It remains to be investigated, if the change in patient management caused by POCUS actually improves patient care, or if it causes harm in terms of false positive findings, misdiagnosis, overdetection and potential, subsequent overtreatment.

\section{CONCLUSION}

POCUS examinations in general practice were used for many different indications and entailed an increased diagnostic reassurance for the GP and a change in diagnosis or management in $71.8 \%$ of patients. The potential high impact of POCUS underlines the need for further research to support an appropriate implementation of POCUS in general practice.

Acknowledgements The authors would like to thank the participating GPs for their individual contributions and the Danish Society for Ultrasound in General Practice (DAUS) for non-financial support of the study.

Contributors All authors participated in designing the study and developing the registration tools. CAA recruited and instructed the participating GPs prior to the study. OG performed the evaluation of the participating GPS POCUS competences. Data collection and analysis were performed by CAA and MBJ. CAA wrote the first draft of the article in collaboration with MBJ. All authors participated in the following review process and contributed to the final version of the article.

Funding This study was conducted as independent research financially supported by the Committee of Multipractice Studies in General Practice and The Danish Research Foundation for General Practice.

\section{Competing interests None declared.}

Patient and public involvement Patients and/or the public were involved in the design, or conduct, or reporting, or dissemination plans of this research. Refer to the Methods section for further details.

Patient consent for publication Not required.

Ethics approval The study was approved by the Danish Data Protection Agency and the Committee of Multipractice Studies. The study was also notified to the Regional Committee on Health Research Ethics, but they responded that their approval was not needed according to Danish law.

Provenance and peer review Not commissioned; externally peer reviewed.

Data availability statement Data are available upon reasonable request. All study data are safely stored at Center for General practice at Aalborg University in Denmark and available upon reasonable request to the corresponding author.

Open access This is an open access article distributed in accordance with the Creative Commons Attribution Non Commercial (CC BY-NC 4.0) license, which permits others to distribute, remix, adapt, build upon this work non-commercially, and license their derivative works on different terms, provided the original work is properly cited, appropriate credit is given, any changes made indicated, and the use is non-commercial. See: http://creativecommons.org/licenses/by-nc/4.0/.

\section{ORCID iDs}

Camilla Aakjær Andersen http://orcid.org/0000-0002-5933-748X

Martin Bach B Jensen http://orcid.org/0000-0003-2162-7390

\section{REFERENCES}

1 Mengel-Jørgensen T, Jensen MB. Variation in the use of point-ofcare ultrasound in general practice in various European countries. Results of a survey among experts. Eur J Gen Pract 2016;22:274-7.

2 Genc A, Ryk M, Suwała M, et al. Ultrasound imaging in the general practitioner's office - a literature review. J Ultrason 2016;16:78-86.

3 Aakjær Andersen C, Jensen MBB, Toftegaard BS, et al. Primary care physicians' access to in-house ultrasound examinations across Europe: a questionnaire study. BMJ Open 2019;9:e030958.

4 Bornemann P, Barreto T. Point-Of-Care ultrasonography in family medicine. Am Fam Physician 2018;98:200.

5 Andersen CA, Holden S, Vela J, et al. Point-Of-Care ultrasound in general practice: a systematic review. Ann Fam Med 2019;17:61-9.

6 Moore CL, Copel JA. Point-Of-Care ultrasonography. N Engl J Med 2011;364:749-57.

7 Dietrich CF, Goudie A, Chiorean L, et al. Point of care ultrasound: a WFUMB position paper. Ultrasound Med Biol 2017;43:49-58. 
8 Diprose W, Verster F, Schauer C. Re-Examining physical findings with point-of-care ultrasound: a narrative review. $N Z$ Med $J$ 2017;130:46-51.

9 Smallwood N, Dachsel M. Point-Of-Care ultrasound (POCUS): unnecessary gadgetry or evidence-based medicine? Clin Med 2018;18:219-24.

10 Laursen CB, Sloth E, Lassen AT, et al. Point-Of-Care ultrasonography in patients admitted with respiratory symptoms: a single-blind, randomised controlled trial. Lancet Respir Med 2014;2:638-46.

11 Gorcsan J, Pandey P, Sade LE. Influence of hand-carried ultrasound on bedside patient treatment decisions for consultative cardiology. $J$ Am Soc Echocardiogr 2004;17:50-5.

12 Greaves K, Jeetley P, Hickman M, et al. The use of hand-carried ultrasound in the hospital setting--a cost-effective analysis. J Am Soc Echocardiogr 2005;18:620-5.

13 Lindelius A, Törngren S, Nilsson L, et al. Randomized clinical trial of bedside ultrasound among patients with abdominal pain in the emergency department: impact on patient satisfaction and health care consumption. Scand J Trauma Resusc Emerg Med 2009;17:60.

14 Colli A, Prati D, Fraquelli M, et al. The use of a pocket-sized ultrasound device improves physical examination: results of an inand outpatient cohort study. PLoS One 2015;10:e0122181.

15 Mumoli N, Vitale J, Giorgi-Pierfranceschi M, et al. General practitioner-performed compression ultrasonography for diagnosis of deep vein thrombosis of the leg: a multicenter, prospective cohort study. Ann Fam Med 2017;15:535-9.

16 Andersen CA, Davidsen AS, Brodersen J, et al. Danish general practitioners have found their own way of using point-of-care ultrasonography in primary care: a qualitative study. BMC Fam Pract 2019;20:89

17 Todsen T, Tolsgaard MG, Olsen BH, et al. Reliable and valid assessment of point-of-care ultrasonography. Ann Surg 2015;261:309-15.

18 The World Organization of Family Doctors' (WONCA) International Classification Committee (WICC). International classification of primary care, 2015. Available: https://www.globalfamilydoctor.com/ site/DefaultSite/filesystem/documents/Groups/WICC/International\% 20 Classification\%20of\%20Primary\%20Care\%20Dec16.pdf [Accessed 14 oct 2019].

19 Praktiserende lægers organisation. PLO FAKTA ARK 2018. [The Danish Medical Association: General Practice Fact-sheet 2018]
2019. Available: https://www.laeger.dk/sites/default/files/plo_ faktaark_2018.pdf [Accessed 7 May 2020].

20 Steinmetz P, Oleskevich S. The benefits of doing ultrasound exams in your office. J Fam Pract 2016;65:517-23.

21 Moth G, Olesen F, Vedsted P. Reasons for encounter and disease patterns in Danish primary care: changes over 16 years. Scand $J$ Prim Health Care 2012;30:70-5.

22 Jalil R, Warren R, Ma IWY, et al. Point of care ultrasound training needs for primary care physicians: practice setting matters. Cogent Education 2019;6:1617826.

23 Bhagra A, Tierney DM, Sekiguchi H, et al. Point-Of-Care ultrasonography for primary care physicians and general internists. Mayo Clin Proc 2016;91:1811-27.

24 American Academy of Family Physicians. Recommended curriculum guidelines for family medicine residents: point of care ultrasound. AAFP reprint No. 290D, 2016. Available: https://www. aafp.org/dam/AAFP/documents/medical_education_residency/ program directors/Reprint290D_POCUS.pdf [Accessed 7 Sep 2020].

25 The Danish College of General Practitioners (DSAM) Ultrasound interest group. Common trunk, 2015. Available: https://www.dsam. dk/flx/organisation/udvalg_og_interessegrupper/ultralyd_i_almen_ praksis/common trunk/ [Accessed 28 Oct 2019].

26 Lindgaard K, Riisgaard L. 'Validation of ultrasound examinations performed by general practitioners'. Scand J Prim Health Care 2017;35:256-61.

27 Pinto A, Pinto F, Faggian A, et al. Sources of error in emergency ultrasonography. Crit Ultrasound J 2013;5 Suppl 1:S1.

28 Olgers TJ, Azizi N, Blans MJ, et al. Point-Of-Care ultrasound (PoCUS) for the internist in acute medicine: a uniform curriculum. Neth J Med 2019;77:168-76.

29 Deshpande R, Akhtar S, Haddadin AS. Utility of ultrasound in the ICU. Curr Opin Anaesthesiol 2014;27:123-32.

30 Shokoohi $\mathrm{H}$, Boniface KS, Zaragoza M, et al. Point-Of-Care ultrasound leads to diagnostic shifts in patients with undifferentiated hypotension. Am J Emerg Med 2017;35:e-1984

31 Shih $\mathrm{CH}$. Effect of emergency physician-performed pelvic sonography on length of stay in the emergency department. Ann Emerg Med 1997;29:348-52.

32 Glasø M, Mediås IB, Straand J. [Diagnostic ultrasound in general practice]. Tidsskr Nor Laegeforen 2007;127:1924-7. 